\title{
Aubrey Sheiham Scholarship established
}

\begin{abstract}
The UCL Dental Public Health Group is pleased to announce the establishment of a new Aubrey Sheiham Scholarship for overseas applicants to the UCL MSc Dental Public Health Programme. The new MSc Scholarship has been established in honour of the late Professor Aubrey Sheiham, an inspirational dental public health scholar, and is funded through very generous personal donations from Dr Garry Rayant and Dr Kathy Fields, and Dr Helena Cronin. In the 2020-21 academic year a $£ 5,000$ Scholarship will be awarded. Further details on how to apply for the Aubrey Sheiham Scholarship can
\end{abstract}

be found at the following link: https://www. ucl.ac.uk/epidemiology-health-care/study/ postgraduate/dental-public-health-msc/ msc-dental-public-health-aubrey-sheihamscholarship.

The UCL Dental Public Health Group, led by Professor Richard Watt, is a WHO Collaborating Centre for Oral Health Inequalities and Public Health. Professor Watt also co-chairs the recently established Lancet Commission on Oral Health.

Further information about the UCL DPH Group can be found at: https://www.ucl. ac.uk/epidemiology-health-care/research/ eph/research/dental-public-health.

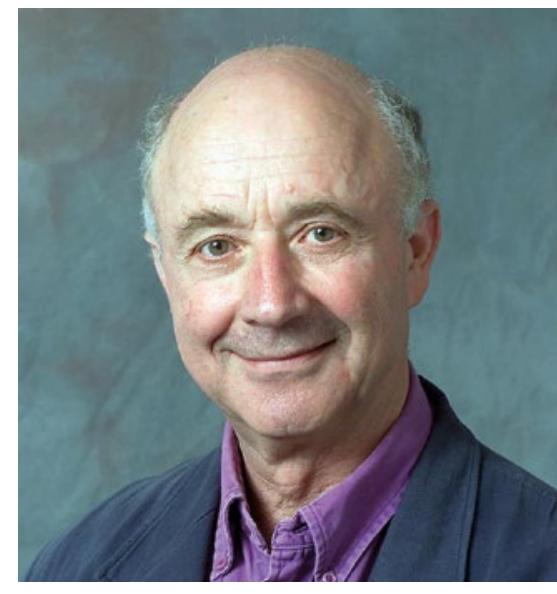

Professor Aubrey Sheiham

\section{Increase in adults seeking orthodontic treatment}

As lockdown restrictions are lifted, many people will be seeking tooth straightening treatment. This is backed up by new figures released by the British Orthodontic Society (BOS) revealing that the number of adults seeking orthodontic treatment in the UK is on the increase [survey carried out online in April].

As the demand for adult orthodontics increases, so do the options for patients. The stats also show concern around the dangers of 'direct to the consumer' teeth straightening - also known as 'DIY braces'. Nearly two thirds of orthodontists surveyed (65\%) are concerned that more patients will be seeking 'DIY braces' both during and after lockdown.

With many practices dealing with a lockdown backlog of patients and members of the public more aware of issues surrounding close contact, there is concern that more people may put themselves in danger with 'DIY' options.

Patients are advised in all

circumstances to visit a trained clinician ensuring that they have the various options open to them explained, in person, so they can make an informed decision. The Safe Brace Campaign www.safebrace.org - is a trusted space where patients can get advice about the safest and most effective way to have orthodontic treatment.

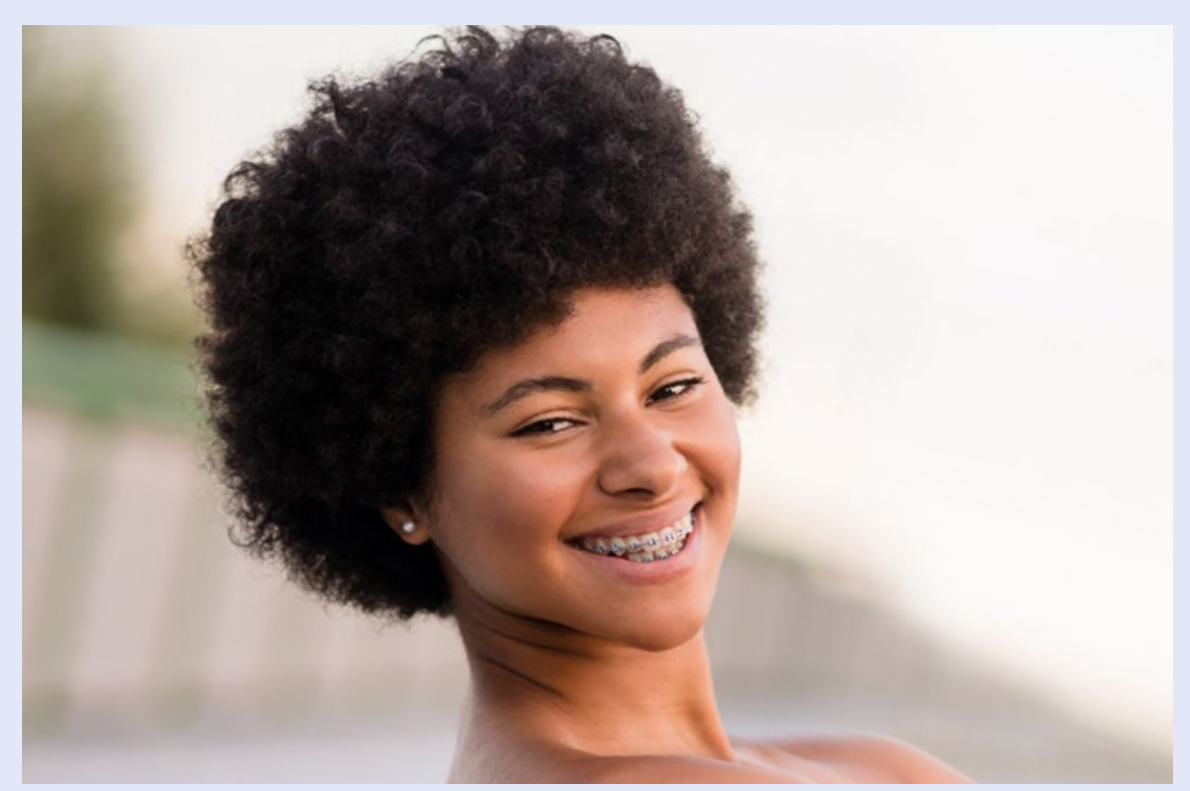

When asked what kind of braces they provide to their patients, orthodontists revealed a cross section of approaches:

- Over $80 \%$ supply fixed braces with clear aesthetic brackets

- Over 30\% supply lingual braces

- $80 \%$ supply clear aligners.

The most popular system, provided by more than $97 \%$ of orthodontists, is fixed braces on the front of the teeth. This figure reflects the high number of young people treated as NHS patients for whom fixed braces is the most appropriate option.
Peter McCallum, BOS Director of External Relations, said: 'It is good to see the number of adults interested in orthodontics remains high; we know that treatment can give people the straight and confident smile they have always dreamed about. We want to ensure patients are given the very best advice about orthodontic treatment. Our members, specialists and dentists with a special interest, offer a range of options for adults, enabling them to provide a solution to any kind of orthodontic problem. 